\title{
A PET Study of Tiagabine Treatment Implicates Ventral Medial Prefrontal Cortex in Generalized Social Anxiety Disorder
}

\author{
Karleyton C Evans*, 1,2, Naomi M Simon', Darin D Dougherty ',2, Elizabeth A Hoge', John J Worthington', \\ Candice Chow', Rebecca E Kaufman', Andrea L Gold', Alan J Fischman², Mark H Pollack' and \\ Scott L Rauch ${ }^{1,3}$ \\ 'Department of Psychiatry, Psychiatric Neuroscience Division, Massachusetts General Hospital, Harvard Medical School, Boston, MA, USA; \\ ${ }^{2}$ Department of Radiology, Massachusetts General Hospital, Harvard Medical School, Boston, MA, USA; ${ }^{3}$ McLean Hospital, Harvard Medical \\ School, Belmont, MA, USA
}

\begin{abstract}
Corticolimbic circuitry has been implicated in generalized social anxiety disorder (gSAD) by several neuroimaging symptom provocation studies. However, there are limited data regarding resting state or treatment effects on regional cerebral metabolic rate of glucose uptake (rCMRglu). Given evidence for anxiolytic effects conferred by tiagabine, a $\gamma$-aminobutyric acid (GABA) reuptake inhibitor, the present $\left[{ }^{18} \mathrm{~F}\right]$ fluorodeoxyglucose-positron emission tomography $\left({ }^{18} \mathrm{FDG}-\mathrm{PET}\right)$ study sought to $(\mathrm{I})$ compare resting rCMRglu between healthy control $(\mathrm{HC})$ and pretreatment gSAD cohorts, (2) examine pre- to post-tiagabine treatment rCMRglu changes in gSAD, and (3) determine rCMRglu predictors of tiagabine treatment response. Fifteen unmedicated individuals with gSAD and ten HCs underwent a baseline (pretreatment) resting-state ${ }^{18}$ FDG-PET scan. Twelve of the gSAD individuals completed an open, 6-week, flexible dose trial of tiagabine, and underwent a second (posttreatment) resting-state ${ }^{18} \mathrm{FDG}-\mathrm{PET}$ scan. Compared to the HC subjects, individuals with gSAD demonstrated less pretreatment rCMRglu within the anterior cingulate cortex and ventral medial prefrontal cortex (vmPFC) at baseline. Following tiagabine treatment, vmPFC rCMRglu increased significantly in the gSAD group. Further, the magnitude of treatment response was inversely correlated with pretreatment rCMRglu within vmPFC. Taken together the present findings converge with neuroimaging findings from studies of social cognition in healthy individuals and symptom provocation in gSAD to support a role for the vmPFC in the pathophysiology of gSAD. Given the pharmacological profile of tiagabine, these findings suggest that its therapeutic effects in gSAD may be mediated by GABAergic modulation within the vmPFC.

Neuropsychopharmacology (2009) 34, 390-398; doi: I 0.I038/npp.2008.69; published online 4 June 2008
\end{abstract}

Keywords: positron emission tomography; social anxiety disorder; tiagabine; prefrontal cortex; neuroimaging

\section{INTRODUCTION}

Social anxiety disorder (SAD) is a common, chronic psychiatric disorder characterized by fear and avoidance of social interactions and public scrutiny. The generalized subtype (gSAD) is associated with significant comorbidity, impairment, and decreased quality of life (Stein, 2006). The pathophysiology underlying SAD is poorly understood. Thus, studies aimed at improving our understanding of the pathophysiology of SAD and treatment-related functional changes are of great clinical importance, particularly given

\footnotetext{
*Correspondence: Dr KC Evans, Department of Psychiatry, Psychiatric Neuroscience Division, Massachusetts General Hospital-East, 13th Street, Building 149, Suite 2625, Charlestown, MA 02129, USA, Tel: + I 617724 0244, Fax: + I 617726 4078,

E-mail: kcevans@partners.org

Previously presented in part as a poster at the 2006 American College of Neuropsychopharmacology Annual Meeting.

Received 20 December 2007; revised 31 March 2008; accepted 7 April 2008
}

the efficacy limitations and side-effect profiles associated with current pharmacotherapy for gSAD (Pollack, 2001; Schwartz et al, 2007).

Comparisons of SAD with healthy control (HC) cohorts during psychiatric neuroimaging studies have revealed neurotransmitter abnormalities (Kent et al, 2002; Phan et al, 2005; Lanzenberger et al, 2007) as well as functional abnormalities within medial prefrontal cortex (eg anterior cingulate cortex, ACC; ventral medial prefrontal cortex, vmPFC) (Lorberbaum et al, 2004; Van Ameringen et al, 2004; Amir et al, 2005; Kilts et al, 2006) and limbic/ paralimbic regions (eg insula, amygdala, hippocampus/ parahippocampal gyrus) (Tillfors et al, 2001; Furmark et al, 2002; Stein et al, 2002; Lorberbaum et al, 2004; Furmark et al, 2005; Straube et al, 2005; Phan et al, 2006) that comprise 'corticolimbic' circuitry. Findings from studies in both animals (Amaral, 2002) and humans (eg lesion, social cognition) (Adolphs, 2003; Frith, 2007; Shamay-Tsoory and Aharon-Peretz, 2007) converge to suggest that this corticolimbic circuitry is vital to social information processing. 
Further, corticolimbic circuitry has been implicated in the genesis of fear/anxiety (Tillfors, 2004). A prevailing canonical corticolimbic model for pathological anxiety suggests that the amygdala and insula, which mediate fear and anxiety responses, are hyper-responsive and inadequately regulated by the vmPFC and hippocampus, structures known to facilitate extinction of conditioned fear responses (Etkin and Wager, 2007; Milad et al, 2007). Support for corticolimbic dysfunction in SAD is garnered from the replication of aberrant amygdalar, insular, and hippocampal findings across several neuroimaging studies. However, convincing evidence for vmPFC dysfunction in SAD remains limited (Etkin and Wager, 2007).

The effects of anxiolytic agents on corticolimbic circuitry in SAD have been examined only recently. For example, the pre-/posttreatment oxygen-15 positron emission tomography (O-15 PET) studies of Furmark et al (2002, 2005) demonstrated attenuated $\mathrm{rCBF}$ in amygdalar and hippocampal regions during symptom provocation following serotonergic, neurokinin antagonist and behavioral treatments of SAD. In contrast, hippocampal increases and no differential amygdalar rCBF following nefazodone treatment were observed by Kilts et al (2006) with O-15 PET to study SAD patients pre-/posttreatment during symptom provocation. The variability across the published O-15 PET studies calls for further investigation of treatment effects on the neural circuitry underlying SAD.

By combining fluorodeoxyglucose $\left({ }^{18} \mathrm{FDG}\right)$-PET with an open treatment trial of the selective $\gamma$-aminobutyric acid (GABA) reuptake inhibitor tiagabine, the present study sought to examine correlates of treatment effects potentially mediated by GABAergic mechanisms in gSAD. Tiagabine increases synaptic availability of GABA via selective inhibition of the GABA transporter-1, which prolongs the effect of endogenous GABA in the synapse (Fink-Jensen et al, 1992; Borden et al, 1994). Open-labeled trials have demonstrated modest support for the efficacy and tolerability of tiagabine for SAD and other anxiety disorders. This support is garnered from studies predating the onset of the present study (Crane, 2003; Rosenthal, 2003; Ninan and Papp, 2004) as well as in more recent studies (Dunlop et al, 2007; Schwartz et al, 2007). The principal objective of the present ${ }^{18}$ FDG-PET study was to test the following hypotheses as related to a model of corticolimbic dysfunction in gSAD: (1) compared to $\mathrm{HC}$ subjects at pretreatment, gSAD patients would exhibit greater regional cerebral metabolic rate of glucose (rCMRglu) uptake in limbic medial temporal lobe regions and lower rCMRglu uptake in ventral prefrontal regions, (2) compared to pretreatment, gSAD patients would demonstrate decreased rCMRglu in limbic medial temporal lobe regions and increased rCMRglu within ventral prefrontal regions following treatment, and (3) the cerebral metabolic profile within these corticolimbic regions of gSAD patients at pretreatment would serve as a predictor of tiagabine treatment response.

\section{METHODS}

\section{Subjects}

Study procedures were approved by the Partners Healthcare Human Research Committee, and all participants received and signed written informed consent. A total of 30 patients were screened to enroll $15 \mathrm{gSAD}$ patients ( 9 men, 6 women) who met entry criteria, completed a baseline scan, and had at least one assessment on tiagabine; $10 \mathrm{HC}$ subjects (6 men, 4 women) were also enrolled (Table 1). All were righthanded, and were without confounding neurological or medical disease. Psychiatric diagnoses were assessed by trained study investigators with the Structured Clinical Interview for DSM-IV (First et al, 1995) to confirm eligibility and examine comorbidity in the gSAD group, as well as to establish the absence of Axis I diagnoses in the HC group. Inclusion criteria for the gSAD group included a

Table I Demographic and Clinical Outcome Data

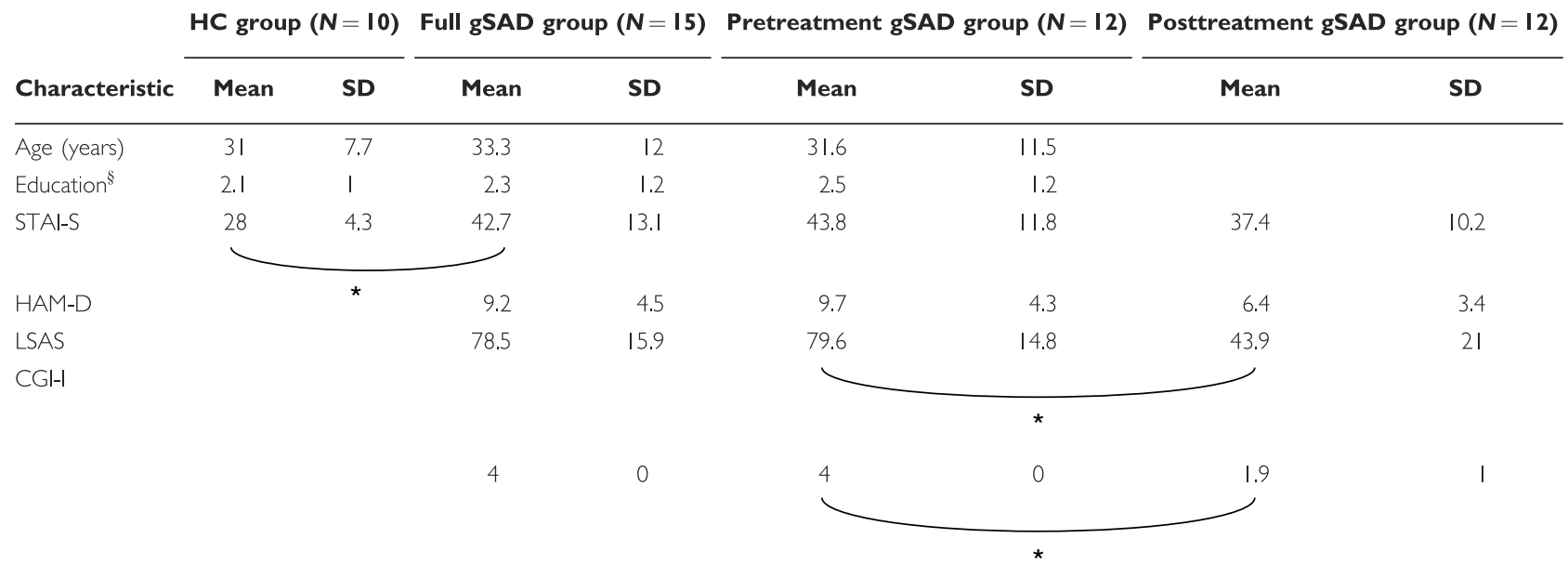

CGI-I, Clinical Global Impression-Improvement Scale; gSAD, generalized social anxiety disorder; HAM-D, Hamilton Depression Scale; HC, healthy control; LSAS,

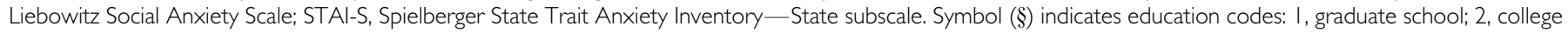
degree; 3, partial college; 4, high school degree; 5, partial high school; 6, junior high school; $7,<7$ years of school. Asterisk indicates between-group differences $(p<0.01)$. 
Liebowitz Social Anxiety Scale (LSAS; Liebowitz, 1987) score $\geqslant 50$ and a primary diagnosis of gSAD, but allowed for secondary comorbid depression and anxiety disorders as long as a Hamilton Depression Scale Score (17-item HAM-D) was $<18$. Current substance use disorders (past 6 months), lifetime psychotic disorders, obsessive-compulsive disorder, and eating disorders were exclusionary.

Among the gSAD cohort, comorbidity was minimal (current: major depressive disorder (MDD) and dysthymia $(N=1)$, generalized anxiety disorder $(N=2)$; past: MDD $(N=3)$, dysthymia $(N=1)$, substance or alcohol use disorder $(N=3))$. All participants were free of psychotropic medication, and those with gSAD had minimal depressive symptoms (Table 1). The mean duration of gSAD was $16.6 \pm 14.1$ years.

\section{Tiagabine Trial}

Open-label tiagabine was initiated at $2 \mathrm{mg}$ per day and then flexibly titrated up $4 \mathrm{mg}$ per week through week 4 then continued to week 6 at a maximum dosage of $16 \mathrm{mg}$ per day. Symptomatic change with tiagabine was assessed with the LSAS and Clinician Global Impression-Improvement (CGI-I) scores. Treatment response was defined as a reduction of $50 \%$ or more on the LSAS at end point. Change in depressive symptoms was assessed with HAM-D scores, and nonspecific anxiety was assessed via the Spielberger's State-Trait Anxiety Index-State (STAI-S; Spielberger et al, 1970) at each PET scan. Safety and tolerability of tiagabine was assessed by recording adverse events and vital signs.

\section{${ }^{18}$ FDG-PET Data Acquisition}

All participants fasted for at least $6 \mathrm{~h}$ prior to arrival to the PET suite. Participants received an intravenous injection of ${ }^{18} \mathrm{FDG}(\sim 370 \mathrm{MBq}$ or $10 \mathrm{mCi}$ ) and remained in a quiet room with eyes open for a 45-min uptake period. Next, each participant's head was immobilized with foam cushions and positioned so that the imaging plane was parallel to the orbitomeatal line. Emission data were acquired with a Siemens HR + PET scanner (CTI Molecular Imaging, Knoxville, TN) for $30 \mathrm{~min}$. The primary imaging parameters of the $\mathrm{HR}+$ scanner are in-plane and axial resolutions of $4.5 \mathrm{~mm}$ full-width half maximum (FWHM) and 63 contiguous slices of 2.5-mm separation. The HR + images were reconstructed using a conventional filtered back-projection algorithm to an in-plane resolution of $4.5 \mathrm{~mm}$ FWHM. Projection data were corrected for nonuniformity of detector response, dead time, random coincidences and scattered radiation. An analytic attenuation correction was applied to the data, based on an estimate of slice contour and the assumption of a uniform attenuation coefficient equal to that of water.

\section{MRI Data Acquisition}

High-resolution MRI data were collected for co-registration with ${ }^{18}$ FDG-PET data. MRI scans were acquired using a Sonata $1.5 \mathrm{~T}$ whole-body high-speed imaging device (Siemens Medical Systems, Iselin, NJ) with a three-axis gradient head coil. After an automated scout image was obtained and shimming procedures were performed, high-resolution, 3D
MPRAGE sequences $\left(\mathrm{TR}=7.25 \mathrm{~ms}, \mathrm{TE}=3 \mathrm{~ms}\right.$, flip angle $=7^{\circ}$ ) were acquired, which generated images with $1 \mathrm{~mm}$ isotropic spatial resolution.

\section{Image Processing and Analysis}

Image preprocessing and analyses were performed following the theory of statistical parametric mapping (SPM) within SPM5 analysis software as reviewed by Friston et al (2007). Pre-processing included motion correction, coregistration, spatial normalization, and smoothing. Specifically, within subjects, posttreatment images were realigned to pretreatment images. Next, each subject's ${ }^{18}$ FDG-PET images were co-registered with their corresponding structural MRI images. Stereotactic spatial normalization of the functional data was performed based on standardized tissue probability templates (Montreal Neurologic Institute (MNI) stereotactic space; http://www.bic.mni.mcgill.ca). Scans were subsequently filtered with a $12-\mathrm{mm}$ FWHM threedimensional Gaussian filter.

After pre-processing, several statistical parametric tests were performed within SPM5 software to test a priori hypotheses related to the corticolimbic model for anxiety. Specifically, voxel-wise tests were performed to identify (1) pretreatment between-group (gSAD $v s \mathrm{HC}$ ) differences in rCMRglu, (2) treatment-related changes in rCMRglu (gSAD posttreatment $v s$ gSAD pretreatment), and (3) gSAD pretreatment cerebral metabolic predictors of tiagabine treatment response. Direct between-group comparisons of gSAD responders (gSAD-R; $\geqslant 50 \%$ change LSAS score) $v s$ gSAD nonresponders (gSAD-NR) were initially considered, but not performed due to insufficient statistical power. Thus, categorical analyses were performed via voxel-wise $t$-tests of pretreatment scans of gSAD-R $v s \mathrm{HC}$ subjects and gSAD-NR vs HC subjects. Peak voxel clusters identified by the gSAD-R vs HC and gSAD-NR vs HC contrasts served as functional regions of interest (ROIs), from which pretreatment rCMRglu was extracted via the MarsBaR SM5-based toolbox (http://marsbar.sourceforge.net) and tested for significant correlation (Pearson's; $p<0.05$ ) with LSAS percent change scores. The planned correlations also included tests for the effects of nonspecific state anxiety and depression via partial correlations using STAI-S and HAM-D percent change scores as regressors. For the cerebral metabolic predictors analyses, both categorical and continuous variable analyses were employed (Evans et al, 2006). The continuous variable analysis was performed first to test for linear regression (correlation) of gSAD pretreatment rCMRglu with LSAS percent change scores. Categorical analyses were performed next.

All statistical parametric maps were created using a liberal significance threshold, $z$-score $\geqslant 3.09 \quad(p<0.001$, uncorrected) and cluster criterion $\geqslant 15$ voxels, by convention, to identify loci of potential statistical significance and spatial extent. Given that the present study represents the first ${ }^{18}$ FDG-PET study in gSAD, relatively liberal statistical thresholds were employed in the hope of establishing more refined hypotheses for future studies. Statistical maps of functional data were inspected with the aid of co-registered structural MRI data. Regional anatomy and Brodmann areas (BAs) were defined using the atlases of Talairach and Tournoux (1988) and Duvernoy (1999) after transformation 
of SPM5 MNI coordinates to Talairach stereotactic coordinates (http://imaging.mrc-cbu.cam.ac.uk/imaging/ mnitalairach). On the basis of findings from prior SAD neuroimaging studies reviewed in 'Introduction', a priori corticolimbic search territories were hypothesized to include the ACC (inclusive of rostral, dorsal, and subcallosal subregions) (Kent et al, 2002; Lorberbaum et al, 2004; Amir et al, 2005; Phan et al, 2005, 2006; Lanzenberger et al, 2007), the insular cortex (Tillfors et al, 2001; Lorberbaum et al, 2004; Straube et al, 2005; Lanzenberger et al, 2007), medial temporal lobe structures (inclusive of amygdala, hippocampus, parahippocampal gyrus) (Tillfors et al, 2001; Furmark et al, 2002, 2005; Stein et al, 2002; Lorberbaum et al, 2004; Straube et al, 2005; Phan et al, 2006), and the vmPFC (Tillfors et al, 2001; Van Ameringen et al, 2004; Kilts et al, 2006; Lanzenberger et al, 2007). Lastly, to obviate bias, the entire brain volume for each voxel-wise statistical parametric map was inspected for other activation loci of comparable significance value, appreciating that the threshold applied ( $p<0.001$, uncorrected) would be unduly liberal to support compelling inferences. However, we only considered such findings as significant in those cases where the actual probability value met the threshold for wholebrain volume correction.

\section{RESULTS}

\section{Tiagabine Trial Dosing, Efficacy, and Tolerability}

Demographic and clinical data are presented in Table 1. The mean gSAD pretreatment LSAS score was $78.5 \pm 15.9$. The mean dose of tiagabine at end point was $12.5 \pm 3.8 \mathrm{mg}$ per day, range $5-16 \mathrm{mg}$ per day. The distribution of tiagabine doses taken was $2-4 \mathrm{mg}(1$ of $15,6.67 \%$ ), 6-8 $\mathrm{mg}$ ( 5 of 15 , $33.33 \%$ ), $10-12 \mathrm{mg}$ ( 2 of $15,13.33 \%$ ), $14-16 \mathrm{mg}$ (7 of 15 , $46.67 \%)$. Twelve patients $(80 \%)$ completed the study. The mean posttreatment reduction in LSAS was $31.07 \pm 19.7$ $(t=6.1$, d.f. $=14, p<0.0001)$ in the intent to treat sample (with at least one visit on tiagabine). Seven of fifteen patients met categorical responder criteria ( $\geqslant 50 \%$ change in LSAS) as well as responder criteria via the secondary efficacy measure of CGI-I (ie score of 1 or 2, 'very much improved' or 'much improved'). Depression as measured by the HAM-D, was unchanged from pre- to posttreatment $(t=1.8$, d.f. $=14$, $p=0.088)$. There was one serious adverse event, but it was deemed unrelated to the study (concussion due to slipping on ice). There were two medication study discontinuations (dissatisfaction with response to tiagabine, and the fall noted above) and one gSAD completer with unusable scan data due to technical issues. Clinical trial results for the $12 \mathrm{gSAD}$ subjects with end point scan data are reported in Table 1. Fourteen of the 15 patients had at least one adverse event, which were all mild to moderate. Adverse events experienced by more than $10 \%$ included sedation (eight), headache (six), dizziness (four), nausea (three), blurred vision (three), fatigue (three), tremor (two), and insomnia (two).

\section{Between-Group Differences in Resting ${ }^{18}$ FDG-PET (gSAD vs HC)}

Compared to the HC group $(N=10)$, the pretreatment gSAD group $(N=15)$ exhibited significantly lower rCMRglu within the left dorsal anterior cingulate cortex (AACC) and left subcallosal cortex (SC; Figure 1; Table 2).

\section{Treatment Effects in gSAD Resting ${ }^{18}$ FDG-PET (Posttreatment vs Pretreatment)}

Compared to pretreatment, gSAD patients with end point scan data $(N=12)$ showed significantly higher rCMRglu at posttreatment (Post $>$ Pre) within the right vmPFC localized to the medial frontal gyrus (Figure 2; Table 2).

\section{Resting ${ }^{18}$ FDG-PET Predictors of Tiagabine Treatment Response in gSAD}

Voxel-wise continuous variable analyses failed to demonstrate a (linear) correlation of gSAD (combined cohort, responders + nonresponders) pretreatment rCMRglu with LSAS percent change scores within a priori hypothesized search territories. However, differential pretreatment rCMRglu was observed in planned categorical voxel-wise $t$-tests. Notably, the gSAD responders (gSAD-R) group $(N=7)$ demonstrated significantly lower rCMRglu within left SC compared to the HC group, whereas the gSAD-NR $(N=5)$ group demonstrated significantly lower rCMRglu within the dACC bilaterally compared to the HC group (Figure 1; Table 2). No significant differences in rCMRglu were observed for the alternative (reverse) contrasts (eg gSAD-R $>\mathrm{HC}$ and gSAD-NR $>\mathrm{HC}$ ). Pretreatment rCMRglu extracted from the SC cluster was found to be inversely correlated with percent LSAS improvement (Pearson's $r=-0.58, p=0.048$, two-tailed; Figure 1). Moreover, tests of partial correlation demonstrated that the significant correlation of pretreatment SC rCMRglu with LSAS percent improvement scores persisted after controlling for STAI-S and HAM-D $(r=-0.66, p=0.037)$. No significant correlation was observed between pretreatment rCMRglu extracted from the dACC cluster with LSAS percent improvement scores (Pearson's $r=-0.29, p=0.355$, two-tailed).

\section{DISCUSSION}

The findings from the present study suggest blunted pretreatment rCMRglu within the $\mathrm{mPFC}$, localized to SC (BA 25) and dACC, indicative of a resting-state metabolic abnormality in gSAD relative to HC individuals. Further, the magnitude of diminished pretreatment SC rCMRglu predicts subsequent improvement in gSAD symptom severity associated with tiagabine treatment. Moreover, relative to pretreatment, a neighboring midline subregion of the vmPFC (BA 10) with comparable $x$ and $z$ coordinates but located slightly more anterior $(y+30 \mathrm{~mm})$ demonstrated a robust increase in rCMRglu following treatment with the GABAergic agent tiagabine. The identified regions (BA 25,10$)$ are known to share dense reciprocal connections within the vmPFC and have been shown to have strong projections to effector sites that mediate conditioned fear responses (eg amygdala, hypothalamus and periaqueductal gray) (Ongur et al, 2003; Quirk et al, 2006). The vmPFC has been recently implicated in fear extinction retention in healthy individuals by both morphometric (Milad et al, 2005) and functional neuroimaging studies (Phelps et al, 2004; Milad et al, 2007). GABA has been proposed to serve 


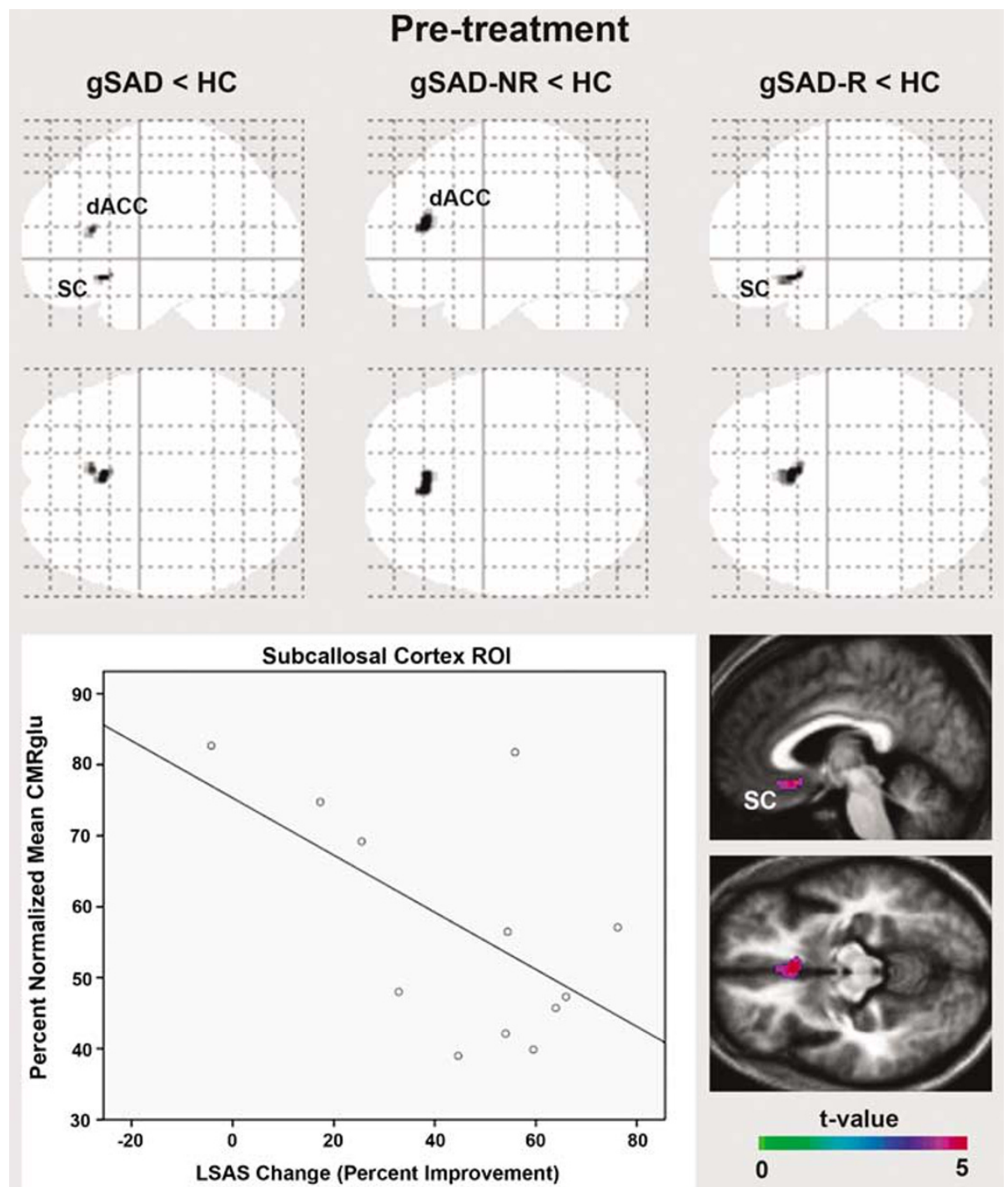

Figure I Upper panel: Glass brain maximum intensity projections for gSAD $<H C$, gSAD-NR $<H C$, and gSAD-R $<H C$ contrasts $(p<0.00$ I, Z-score $\geqslant 3.09$, cluster threshold 15 voxels). gSAD, generalized social anxiety disorder (entire pretreatment cohort, $N=15$ ); gSAD-NR, treatment nonresponder $(N=5)$; gSAD-R, treatment responder $(N=7)$; $H C$, healthy control $(N=10)$; dACC, dorsal anterior cingulate cortex; $S C$, subcallosal cortex. Lower panel, right: Functional data overlaid onto gSAD mean structural image. Higher $t$-scores on the color scale indicate a greater magnitude of diminished pretreatment rCMRglu for gSAD-R <HC contrast. Lower panel, left: Analysis of pretreatment rCMRglu extracted from SC cluster (gSAD-R $<H C$ contrast; $x=-4, y=\mid 8$, $z=-10$ ) identified an inverse correlation with LSAS score percent improvement (Pearson product; $r=-0.58 ; p=0.048$, two-tailed).

Table 2 Local Maxima Reported in Talairach Coordinate System (mm)

\begin{tabular}{|c|c|c|c|c|c|c|c|c|c|c|}
\hline Contrast & gSAD group & Region & BA & Side & $\mathbf{x}$ & $y$ & $\mathbf{z}$ & $\mathbf{T}$ & z-score & Voxels \\
\hline \multirow[t]{4}{*}{ gSAD Pre $<\mathrm{HC}$} & $\mathrm{R}+\mathrm{NR}$ & SC & 25 & $L$ & -4 & 18 & -10 & 4.11 & 3.48 & 17 \\
\hline & $\mathrm{R}+\mathrm{NR}$ & $\mathrm{dACC}$ & 24 & $L$ & -8 & 26 & 15 & 3.85 & 3.31 & 23 \\
\hline & NR & $\mathrm{dACC}$ & 24,32 & $\mathrm{R}$ & 3 & 32 & 19 & 5.72 & 3.82 & 155 \\
\hline & $N R$ & $\mathrm{dACC}$ & 24,32 & $L$ & -3 & 30 & 21 & 5.61 & 3.78 & \\
\hline
\end{tabular}

$P<0.001 ;$ cluster threshold $\geqslant 15$ voxels.

BA, Brodmann area; gSAD, generalized social anxiety disorder (entire cohort, $N=15)$; gSAD-NR, treatment nonresponder $(N=5)$; gSAD-R, treatment responder $(N=7) ; H C$, healthy control $(N=10)$; Pre, pretreatment; Post, post-treatment; dACC, dorsal anterior cingulate cortex; SC, subcallosal cortex; vmPFC, ventral medial prefrontal cortex; L, left; R, right. No significant voxels for gSAD $>H C$ or Post $<$ Pre contrasts. 
as the primary neurotransmitter within the corticolimbic circuitry-mediating fear extinction (Quirk et al, 2006; Akirav and Maroun, 2007) and GABAergic agents have demonstrated clinical efficacy across the anxiety disorders (Lydiard, 2003). Social stress has been shown to reduce

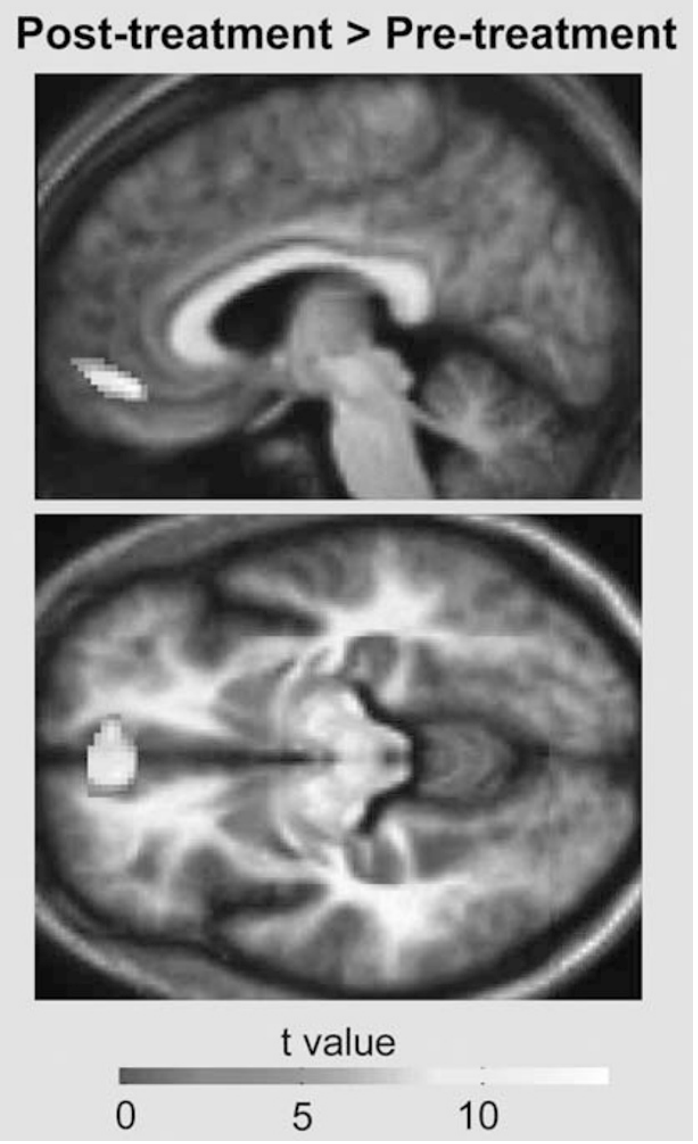

Figure 2 Increase in rCMRglu within bilateral vmPFC of gSAD patients $(N=12)$ for Post-treatment $>$ Pretreatment contrast $(p<0.00 \mathrm{I}$, Z-score $\geqslant 3.09$, cluster threshold $\geqslant 15$ voxels). Functional data overlaid onto gSAD mean structural image. Higher $t$-scores on the gray scale indicate a greater magnitude of post-treatment rCMRglu. Local maxima (543-voxel cluster) coordinates; $x=3, y=47, z=-7$.
GABA receptor function in rats (Serra et al, 2000), whereas direct infusion of GABA agonists to the rat infralimbic cortex (vmPFC homologue) has been demonstrated to facilitate fear extinction (Akirav and Maroun, 2007). Taken together, the present findings of blunted rCMRglu within vmPFC at pretreatment followed by enhanced vmPFC rCMRglu post-GABAergic treatment are well in accord with the other translational evidence supporting a significant role for GABA within the prevailing corticolimbic model for pathological anxiety/fear.

In a recent anxiety neuroimaging meta-analysis, Etkin and Wager (2007) identified blunted vmPFC activity as a common finding in studies of PTSD, but uncommon to studies of SAD. We are aware of only three neuroimaging studies in SAD patients with vmPFC findings comparable to those in the present study (Table 3). Both Tillfors et al (2001) and Van Ameringen et al (2004) observed reduced rCBF in SAD patients during symptom provocation at loci in close proximity to the posttreatment vmPFC locus observed in the present study (Table 3). Using O-15 PET during a pre-/posttreatment symptom provocation study in SAD patients, Kilts et al (2006) observed nefazodone treatment-related increases in $\mathrm{rCBF}$ within two medial frontal regions comparable to the present study (Table 3). Finally, the findings of Furmark et al (2002) must be acknowledged as they appear to contradict those of the present study as well as the other studies mentioned (Table 3). In contrast to posttreatment increases in vmPFC activity, Furmark et al (2002) reported posttreatment decreases in subcallosal rCBF in SAD citalopram responders during an O-15 PET pre-/posttreatment symptom provocation study. Discordance in subcallosal findings of Furmark et al (2002) with the present study may be due to methodological differences such as: (1) heterogeneous SAD $v s$ exclusive gSAD cohorts, (2) serotonergic $v s$ GABAergic treatment, and/or (3) symptom provocation $v s$ resting-state paradigms. Moreover, emerging evidence indicates significant interaction between serotonergic and GABAergic systems within the PFC (Santana et al, 2004; Lanzenberger et al, 2007). In sum, the vmPFC findings of the present study meaningfully extend and complement previous findings in SAD.

Table 3 Local Maxima Reported in Talairach Coordinate System (mm)

\begin{tabular}{|c|c|c|c|c|c|c|c|}
\hline Region & Study & Contrast & $\Delta$ rCMRglu or $\Delta$ rCBF & Side & $\mathbf{x}$ & $y$ & $\mathbf{z}$ \\
\hline \multirow[t]{3}{*}{ SC } & Present Study & gSAD Pre $<\mathrm{HC}$ & $\downarrow$ & L & -4 & 18 & -10 \\
\hline & Kilts et al (2006) & gSAD Prov Post > gSAD Prov Pre & $\uparrow$ & L & -4 & 23 & -13 \\
\hline & Furmark et al (2002) & gSAD Prov Post > gSAD Prov Pre & $\downarrow$ & $\mathrm{L}$ & -4 & 27 & -7 \\
\hline & Van Ameringen et al (2004) & gSAD Prov $>$ gSAD CT & $\downarrow$ & $\mathrm{R}$ & 4 & 37 & -9 \\
\hline & Tillfors et al (200I) & SAD Prov $<$ HC Prov & $\downarrow$ & $\mathrm{R}$ & 3 & 58 & -9 \\
\hline
\end{tabular}

BA, Brodmann area; gSAD, generalized social anxiety disorder; HC, healthy control; Pre, pretreatment; Post, post-treatment; Prov, provocation; CT, control task; SC, subcallosal cortex; vmPFC, ventral medial prefrontal cortex; $\Delta$ rCMRglu, difference in regional cerebral metabolic rate of glucose uptake; $\Delta$ rCBF, difference in regional cerebral blood flow; $\uparrow$, increase; $\downarrow$, decrease; L, left; R, right; — indicates that authors provided graphical representation of published data within the indicated region without Talairach coordinates. 
Early case reports of patients with prefrontal lesions (eg patient Phineas Gage) have demonstrated human reliance on prefrontal integrity for adequate and appropriate social functioning (Harlow, 1868). More recent human lesion studies have shown vmPFC specificity for certain types of socio-affective processing. Shamay-Tsoory and AharonPeretz (2007) found that compared to patients with lesions in other locations (eg ventrolateral PFC), patients with vmPFC lesions demonstrated greater impairment in their ability to make judgments about the affective states of others. Neuroimaging studies of healthy individuals complement lesion studies, as the medial PFC has been consistently implicated during social information-processing tasks (Adolphs, 2003; Frith, 2007). More specifically, the vmPFC has been implicated in emotional valence discrimination of social scenes and faces (Geday et al, 2003), self-referential processing, as well as making inferences of the mental states of others relative to self (Mitchell et al, 2006). Thus, converging lines of evidence appear to support a role for vmPFC in socio-affective processing. As socio-affective neuroimaging probes specifically designed to test vmPFC function have yet to be studied in SAD, we speculate that the significance of the present findings of enhanced metabolism within the vmPFC at posttreatment may reflect improved socio-affective information processing.

Beyond the vmPFC, the present study also identified aberrant metabolism within the dACC. Relative to the HC cohort, the entire gSAD cohort $(N=15)$ demonstrated pretreatment rCMRglu blunting within the left dACC (Table 2; Figure 1). Further, subgroup analyses revealed the relative bias of the gSAD-NR cohort contribution to the combined cohort pretreatment map, as the gSAD-NR cohort, but not gSAD-R cohort, demonstrated bilateral blunting of rCMRglu within the dACC territory when compared to the HC cohort. In contrast to the vmPFC and SC findings discussed above, ACC findings across functional neuroimaging studies of SAD have been fairly inconsistent (Furmark et al, 2002; Lorberbaum et al, 2004; Amir et al, 2005; Kilts et al, 2006). We speculate that these differences are related to the sources of variance mentioned earlier (eg SAD sample heterogeneity, pharmacology, scanning state). Despite the differences, the consistency of dACC location should be underscored and awaits further investigation.

Interpretations of the present study should be considered in the context of acknowledged limitations. As the average placebo response rate across randomized controlled trials in SAD has been reported to be approximately 30\% (Ipser et al, 2008), the measured differential brain activity associated with treatment response in the present study could reflect a combination of medication and expectancy/ placebo responses inherent to the open pharmacotherapy trial. This limitation may be mitigated partially by the fact that the pre- to posttreatment midline vmPFC finding observed in the present study is anatomically distinct from a common mediating anatomy that has been observed for placebo responses in pain, depression, and anxiety (eg dorsolateral/ventrolateral PFC, rostral and dorsal ACC) (Mayberg et al, 2002; Benedetti et al, 2005). The region of SC identified by the present study as a treatment predictor (lower pretreatment rCMRglu in responders) has however been implicated in placebo responses, yet the direction of change for rCMRglu within SC in the present study is opposite to posttreatment rCMRglu decreases observed in placebo responses (Mayberg et al, 2002). Even so, we cannot exclude the possibility that the present findings, in part, represent placebo responses, as a placebo-response circuitry specific to $\mathrm{SAD}$ has yet to be established. The relatively small patient cohort serves as another important limitation as inferences from the present study to other clinical gSAD samples should be considered with caution. However, the clinical trial described in the present study is comparable to other neuroimaging treatment studies in SAD (Kilts et al, 2006). The clinical trial component of the present study may be best viewed as a vehicle to illustrate the important pathophysiological findings identified. The statistical threshold applied may be viewed as somewhat liberal but was deemed appropriate for this first ever ${ }^{18}$ FDG-PET study of gSAD, emphasizing the necessity of future studies to assess the reproducibility and generalizability of the present findings. Lastly, in contrast to most previous neuroimaging studies of SAD that employed symptom provocation or cognitive activation paradigms (Etkin and Wager, 2007), or symptom provocation combined with pre-/posttreatment paradigms (Furmark et al, 2002, 2005; Kilts et al, 2006), no differential activity within the amygdala or associated medial temporal lobe structures was identified in the present study of resting-state glucose metabolism. Analytic differences such as the voxel-wise approach employed by the present study, in contrast to the ROI approach employed by Furmark et al $(2002,2005)$ may have contributed to the differences in amygdalar findings. However, there was no evidence of differential activity within the medial temporal lobe territories in any of the planned voxel-wise analysis, even at a relaxed threshold of $z$-score $\geqslant 2.58(p<0.005$, uncorrected $)$. Further, the temporal resolution afforded by FDG-PET (approximately $20 \mathrm{~min}$ uptake) almost certainly precluded the detection of exaggerated amygdalar responses (Breiter et al, 1996). Although temporal resolution in this context may be viewed as a limitation, the potential for FDG-PET to identify resting-state between-group abnormalities and pre-/posttreatment changes should be underscored as this method has served as an invaluable tool for informing the pathophysiology underlying several conditions across the mood and anxiety disorders (eg MDD and obsessive compulsive disorder; Evans et al, 2006).

In summary, study results across three different analyses converge to suggest a possible role for the vmPFC in the pathophysiology and treatment response of gSAD. The present vmPFC findings converge with prior neuroimaging findings from studies of social cognition in healthy individuals and of symptom provocation in SAD. The present findings also bear common functional correlates with earlier studies examining human vmPFC lesions. Taken together, the present findings provide additional support for a corticolimbic model of SAD pathophysiology. In addition, this study's findings offer preliminary support for the potential strategy of using neuroimaging methods to predict treatment response and thus improve the clinical management of SAD, as well as a step toward identifying the putative neural circuitry underlying this common yet serious disease. 


\section{ACKNOWLEDGEMENTS}

The study was supported by a grant from Cephalon Inc. Dr Evans was supported by the Clinical Investigator Training Program (Harvard/MIT/HST-BIDMC, in collaboration with Pfizer Inc. and Merck \& Co Inc.), the American Psychiatric Research Institute for Research and Education/Merck \& Co Early Academic Career Research Award and NIH grants MH T32 19126 and EB T32 1632-03. Dr Rauch was supported in part by NIMH R01 grants MH60219, MH074848, and MH070730. We thank the research participants as well as Diana Fischmann, Elizabeth Scannell, and Steve Weise, for technical assistance.

\section{DISCLOSURES/CONFLICTS OF INTEREST}

This study was supported by an investigator-initiated grant from Cephalon. Additional disclosures from the individual authors are listed below.

\section{Karleyton C Evans}

Participation in sponsored clinical trials: Cephalon, Cyberonics, Northstar Neuroscience, Pfizer

Unrestricted research fellowship support: Janssen, Merck, Pfizer

\section{Naomi M Simon}

Participation in sponsored clinical trials: NIMH, NARSAD, Astra Zeneca, Cephalon, Forest Laboratories, GlaxoSmithKline, Lilly, Pfizer, UCB-Pharma, Sepracor

Advisory Boards and Consulting: Solvay

\section{Darin D Dougherty}

Participation in sponsored clinical trials: McNeil, Cyberonics, Forest Laboratories, Lilly, Medtronics, Northstar Neuroscience

Speaker's Bureau for CME and other presentations: Cyberonics, McNeil

\section{Elizabeth A Hoge}

Participation in sponsored clinical trials: Astra Zeneca, Cephalon, Forest Laboratories, GlaxoSmithKline, Janssen, Lilly, Pfizer, UCB-Pharma, Sepracor

Unrestricted research fellowship support: Merck, Pfizer John J Worthington

Participation in sponsored clinical trials: Abbott Laboratories, Alkermes, Aspect Medical Systems, Astra-Zeneca, Bristol-Myers Squibb Company, Cephalon, Eli Lilly, Forest Pharmaceuticals Inc., GlaxoSmithkline, J \& J Pharmaceuticals, Lichtwer Pharma GmbH, Lorex Pharmaceuticals, Novartis, Organon Inc., PamLab, LLC, Pfizer Inc., Pharmavite, Roche, Sanofi-Aventis, Sepracor, Solvay Pharmaceuticals Inc., UCB Pharma and Wyeth-Ayerst Laboratories

Speaker's Bureau for CME and other presentations: Bristol-Myers Squibb Company, Eli Lilly, Forest Pharmaceuticals Inc., GlaxoSmithKline, Pfizer Inc., Sanofi-Aventis and Wyeth-Ayerst Laboratories

Candice Chow

No financial interests to disclose.

Rebecca E Kaufman

No financial interests to disclose.

Andrea L Gold

Participation in sponsored clinical trials: Cephalon

Alan J Fischman

Participation in sponsored clinical trials: Cephalon, GlaxoSmithKline, McNeil Inc., Shire Pharmaceuticals Group
Mark H Pollack

Advisory Boards and Consulting: AstraZeneca, Brain Cells Inc., Bristol Myers Squibb, Cephalon, Forest Laboratories, GlaxoSmithKline, Janssen, Jazz Pharmaceuticals, Eli Lilly, Medavante, Neurocrine, Neurogen, Novartis, Otsuka Pharmaceuticals, Pfizer, Predix, Roche, Sanofi, Sepracor, Solvay, Tikvah Therapeutics, Transcept Inc., UCB Pharma, Wyeth

Research Grants: Bristol Myers Squibb, Cephalon, Forest Laboratories, GlaxoSmithKline, Janssen, Eli Lilly, NARSAD, NIDA NIMH, Pfizer, Sepracor, UCB Pharma, Wyeth

Speaker's Bureau for CME and other presentations: Bristol Myers Squibb, Forest Laboratories, GlaxoSmithK-

line, Janssen, Lilly, Pfizer, Solvay, Wyeth

Equity: Medavante, Mensante Corporation

Scott L Rauch

Consulting: Novartis, Neurogen, Sepracor

Participation in sponsored clinical trials: Cephalon, Cyberonics, Medtronic Inc.

Speaker's Bureau for CME and other presentations: Novartis

Unrestricted research support of laboratory fellows: Pfizer

\section{REFERENCES}

Adolphs R (2003). Cognitive neuroscience of human social behaviour. Nat Rev Neurosci 4: 165-178.

Akirav I, Maroun M (2007). The role of the medial prefrontal cortex-amygdala circuit in stress effects on the extinction of fear. Neural Plast: doi:10.1155/2007/30873.

Amaral DG (2002). The primate amygdala and the neurobiology of social behavior: implications for understanding social anxiety. Biol Psychiatry 51: 11-17.

Amir N, Klumpp H, Elias J, Bedwell JS, Yanasak N, Miller LS (2005). Increased activation of the anterior cingulate cortex during processing of disgust faces in individuals with social phobia. Biol Psychiatry 57: 975-981.

Benedetti F, Mayberg HS, Wager TD, Stohler CS, Zubieta JK (2005). Neurobiological mechanisms of the placebo effect. J Neurosci 25: 10390-10402.

Borden LA, Murali Dhar TG, Smith KE, Weinshank RL, Branchek TA, Gluchowski C (1994). Tiagabine, SK\&F 89976-A, CI-966, and NNC-711 are selective for the cloned GABA transporter GAT-1. Eur J Pharmacol 269: 219-224.

Breiter HC, Etcoff NL, Whalen PJ, Kennedy WA, Rauch SL, Buckner RL et al (1996). Response and habituation of the human amygdala during visual processing of facial expression. Neuron 17: $875-887$.

Crane D (2003). Tiagabine for the treatment of anxiety. Depress Anxiety 18: 51-52.

Dunlop BW, Papp L, Garlow SJ, Weiss PS, Knight BT, Ninan PT (2007). Tiagabine for social anxiety disorder. Hum Psychopharmacol 22: 241-244.

Duvernoy H (1999). The Human Brain. Surface, Three Dimensional Sectional Anatomy with MRI, and Blood Supply. Springer-Wien: New York.

Etkin A, Wager TD (2007). Functional neuroimaging of anxiety: a meta-analysis of emotional processing in PTSD, social anxiety disorder, and specific phobia. Am J Psychiatry 164: 1476-1488.

Evans KC, Dougherty DD, Pollack MH, Rauch SL (2006). Using neuroimaging to predict treatment response in mood and anxiety disorders. Ann Clin Psychiatry 18: 33-42.

Fink-Jensen A, Suzdak PD, Swedberg MD, Judge ME, Hansen L, Nielsen PG (1992). The gamma-aminobutyric acid (GABA) uptake inhibitor, tiagabine, increases extracellular brain levels of GABA in awake rats. Eur J Pharmacol 220: 197-201. 
First MB, Spitzer RL, Gibbon M, Williams JB (1995). Structured Clinical Interview for DSM-IV-TR Axis I Disorders. SCID-I/P. New York State Psychiatric Institute: New York. Research Version.

Friston KJ, Ashburner J, Kiebel SJ, Nichols TE, Penny WD (2007). Statistical Parametric Mapping the Analysis of Functional Brain Images. Academic Press: London.

Frith CD (2007). The social brain? Philos Trans $R$ Soc Lond B Biol Sci 362: 671-678.

Furmark T, Appel L, Michelgard A, Wahlstedt K, Ahs F, Zancan S et al (2005). Cerebral blood flow changes after treatment of social phobia with the neurokinin-1 antagonist GR205171, citalopram, or placebo. Biol Psychiatry 58: 132-142.

Furmark T, Tillfors M, Marteinsdottir I, Fischer H, Pissiota A, Langstrom B et al (2002). Common changes in cerebral blood flow in patients with social phobia treated with citalopram or cognitive-behavioral therapy. Arch Gen Psychiatry 59: 425-433.

Geday J, Gjedde A, Boldsen AS, Kupers R (2003). Emotional valence modulates activity in the posterior fusiform gyrus and inferior medial prefrontal cortex in social perception. Neuroimage 18: 675-684.

Harlow JM (1868). Recovery from the passage of an iron bar through the head. Pub Mass Med Soc 2: 239-347.

Ipser JC, Kariuki CM, Stein DJ (2008). Pharmacotherapy for social anxiety disorder: a systematic review. Expert Rev Neurother 8: 235-257.

Kent JM, Mathew SJ, Gorman JM (2002). Molecular targets in the treatment of anxiety. Biol Psychiatry 52: 1008-1030.

Kilts CD, Kelsey JE, Knight B, Ely TD, Bowman FD, Gross RE et al (2006). The neural correlates of social anxiety disorder and response to pharmacotherapy. Neuropsychopharmacology 31: 2243-2253.

Lanzenberger RR, Mitterhauser M, Spindelegger C, Wadsak W, Klein N, Mien LK et al (2007). Reduced serotonin-1A receptor binding in social anxiety disorder. Biol Psychiatry 61: 1081-1089.

Liebowitz MR (1987). Social phobia. Mod Probl Pharmacopsychiatry 22: 141-173.

Lorberbaum JP, Kose S, Johnson MR, Arana GW, Sullivan LK, Hamner MB et al (2004). Neural correlates of speech anticipatory anxiety in generalized social phobia. Neuroreport 15: 2701-2705.

Lydiard RB (2003). The role of GABA in anxiety disorders. J Clin Psychiatry 64: 21-27.

Mayberg HS, Silva JA, Brannan SK, Tekell JL, Mahurin RK, McGinnis S et al (2002). The functional neuroanatomy of the placebo effect. Am J Psychiatry 159: 728-737.

Milad MR, Quinn BT, Pitman RK, Orr SP, Fischl B, Rauch SL (2005). Thickness of ventromedial prefrontal cortex in humans is correlated with extinction memory. Proc Natl Acad Sci USA 102: 10706-10711.

Milad MR, Wright CI, Orr SP, Pitman RK, Quirk GJ, Rauch SL (2007). Recall of fear extinction in humans activates the ventromedial prefrontal cortex and hippocampus in concert. Biol Psychiatry 62: 446-454.

Mitchell JP, Macrae CN, Banaji MR (2006). Dissociable medial prefrontal contributions to judgments of similar and dissimilar others. Neuron 50: 655-663.

Ninan PT, Papp LA (2004). The Effects of Tiagabine in Patients with Social Anxiety Disorder. Anxiety Disorders Association of America Annual Conference: Miami, FL.
Ongur D, Ferry AT, Price JL (2003). Architectonic subdivision of the human orbital and medial prefrontal cortex. J Comp Neurol 460: 425-449.

Phan KL, Fitzgerald DA, Cortese BM, Seraji-Bozorgzad N, Tancer ME, Moore GJ (2005). Anterior cingulate neurochemistry in social anxiety disorder: $1 \mathrm{H}-\mathrm{MRS}$ at 4 Tesla. Neuroreport 16: 183-186.

Phan KL, Fitzgerald DA, Nathan PJ, Tancer ME (2006). Association between amygdala hyperactivity to harsh faces and severity of social anxiety in generalized social phobia. Biol Psychiatry 59: 424-429.

Phelps EA, Delgado MR, Nearing KI, LeDoux JE (2004). Extinction learning in humans: role of the amygdala and vmPFC. Neuron 43: 897-905.

Pollack MH (2001). Comorbidity, neurobiology, and pharmacotherapy of social anxiety disorder. J Clin Psychiatry 62: 24-29.

Quirk GJ, Garcia R, Gonzalez-Lima F (2006). Prefrontal mechanisms in extinction of conditioned fear. Biol Psychiatry 60: 337-343.

Rosenthal M (2003). Tiagabine for the treatment of generalized anxiety disorder: a randomized, open-label, clinical trial with paroxetine as a positive control. J Clin Psychiatry 64: 1245-1249.

Santana N, Bortolozzi A, Serrats J, Mengod G, Artigas F (2004). Expression of serotonin1A and serotonin2A receptors in pyramidal and GABAergic neurons of the rat prefrontal cortex. Cereb Cortex 14: 1100-1109.

Schwartz TL, Nasra GS, Ashton AK, Kang D, Kumaresan H, Chilton $\mathrm{M}$ et al (2007). An open-label study to evaluate switching from an SSRI or SNRI to tiagabine to alleviate antidepressantinduced sexual dysfunction in generalized anxiety disorder. Ann Clin Psychiatry 19: 25-30.

Serra M, Pisu MG, Littera M, Papi G, Sanna E, Tuveri F et al (2000). Social isolation-induced decreases in both the abundance of neuroactive steroids and $\mathrm{GABA}(\mathrm{A})$ receptor function in rat brain. $J$ Neurochem 75: 732-740.

Shamay-Tsoory SG, Aharon-Peretz J (2007). Dissociable prefrontal networks for cognitive and affective theory of mind: A lesion study. Neuropsychologia 45: 3054-3067.

Spielberger CD, Gorusch RL, Lushene RE (1970). Manual for the State-Trait Anxiety Inventory. Consulting Psychologists Press: Palo Alto, CA.

Stein MB (2006). An epidemiological perspective on social anxiety disorder. J Clin Psychiatry 67: 3-8.

Stein MB, Goldin PR, Sareen J, Zorrilla LT, Brown GG (2002). Increased amygdala activation to angry and contemptuous faces in generalized social phobia. Arch Gen Psychiatry 59: 1027-1034.

Straube T, Mentzel HJ, Miltner WH (2005). Common and distinct brain activation to threat and safety signals in social phobia. Neuropsychobiology 52: 163-168.

Talairach J, Tournoux P (1988). Coplanar Stereotaxic Atlas of the Human Brain. Thieme: Stuttgart.

Tillfors M (2004). Why do some individuals develop social phobia? A review with emphasis on the neurobiological influences. Nord J Psychiatry 58: 267-276.

Tillfors M, Furmark T, Marteinsdottir I, Fischer H, Pissiota A, Langstrom B et al (2001). Cerebral blood flow in subjects with social phobia during stressful speaking tasks: a PET study. Am J Psychiatry 158: 1220-1226.

Van Ameringen M, Mancini C, Szechtman H, Nahmias C, Oakman JM, Hall GB et al (2004). A PET provocation study of generalized social phobia. Psychiatry Res 132: 13-18. 